\title{
Hip fracture in centenarians: Retrospective analysis of an orthogeriatric unit reveals the limitations of the current research
}

\author{
Paolo Mazzola $^{1,2 *}$, Sabrina Perego ${ }^{1}$, Domenico Picone ${ }^{1}$, Maurizio Corsi ${ }^{1}$, Eleni Tsiantouli ${ }^{1}$, Francesco De Filippi ${ }^{3}$, Giuseppe Castoldi ${ }^{4}$, Giuseppe \\ Bellelli $^{1,2}$, Giovanni Zatti ${ }^{5}$ and Giorgio Annoni ${ }^{1,2 *}$ \\ ${ }^{1}$ Department of Health Sciences, University of Milano-Bicocca, and Geriatric Clinic, San Gerardo University Hospital, 20900 Monza, MB, Italy \\ ${ }^{2}$ NeuroMI, Milan Center for Neuroscience, Clinical Neurosciences research area, 20126 Milano, MI, Italy \\ ${ }^{3}$ Department of Medicine, Geriatric Rehabilitation and Sub-acute Care Unit, Regional Hospital of Valtellina and Valchiavenna, 23100 Sondrio, SO, Italy \\ ${ }^{4}$ Department of Orthopedics and Traumatology, Hospital of Giussano, 20833 Giussano, MB, Italy \\ ${ }^{5}$ Department of Surgery, University of Milano-Bicocca, and Orthopaedic Clinic, San Gerardo University Hospital, 20900 Monza, MB, Italy
}

\begin{abstract}
Life expectancy of Italian people is among the highest in the world, at 80 years for males and 85 years for females. Estimates from the National Institute of Statistics show that people aged 65 years and older represent more than $21 \%$ of the Italian population. Among them, centenarians represent the age group with the highest growing rate. Hip fracture incidence increases with advancing age, and currently represents one of the major causes of hospitalization in the elderly. Many studies regarding hip fracture in the elderly have been published, but research focusing on centenarians is still very limited despite their growing trend. This manuscript reviews the activity of the Orthogeriatric Unit at San Gerardo University Hospital (Northern Italy) from its establishment in 2007 until the present, and enlightens the limitations of the research in this area reviewing the literature currently available.
\end{abstract}

Over a 7-year period, we admitted a small population of 10 centenarian female patients, all of whom underwent surgery. Their in-hospital (1 out of 10) and 1-year mortality rates (8 out of 10 ) are consistent with the existing literature.

Centenarians pose an increasing challenge to physicians and surgeons. Rates of recovery of the pre-fracture condition are undoubtedly lower than those in younger geriatric patients, but surgery and early rehabilitation should not be denied solely due to the extreme age of these subjects. However, the research in this area presents several limitations up to now. The available studies are sparse, describe small populations, and are generally a single-center retrospective review of the local hospital admissions registry. Moreover, the objectives and outcome measurements vary considerably between studies, making a statistical comparison unfeasible.

In this scenario, the implementation and analysis of large-scale databases would allow appropriate research among centenarians, including cost-effectiveness of specific surgical treatments.

\section{Introduction}

Life expectancy at birth in Italy is on average 83.1 years $(80.2$ for men and 85.0 years for women), making this country one of the 10 topranked nations in the world for longevity (World Health Organization 2014 report, available at (http://www.who.int/mediacentre/news/ releases/2014/world-health-statistics-2014/en/). Italy's population is one of the oldest in the world, with an expected increase in the proportion of the geriatric population (aged $\geq 65$ years) of $20.9 \%$ to 21.5\% during 2013-2015 (available at http://demo.istat.it). Within this population, individuals aged $\geq 100$ years old represent a particular subgroup. Reaching very advanced age can be considered an example of successful aging, although this homeostasis is frail [1]. Indeed, it is known that the process of aging gradually results in a reduction of the functional capacity of organs. In this age group, referred to as centenarians, the greatly reduced functional organ reserve represents a risk factor for global decline and death, because any stressor can potentially lead to severe organ dysfunction. Fractures and surgical operations are very stressful experiences, and the consequences of such events on a centenarian subject could be devastating [1]. Besides high mortality rates, disability is also a common sequela of major fractures (especially hip fractures) in this age group [2,3].

The Italian National Institute of Statistics (ISTAT, http://www.istat. it/) estimated that the total number of centenarians in Italy was 20,331 individuals in 2014, which is among the highest in Europe (available at http://demo.istat.it), and that they represent the subgroup with the

Correspondence to: Paolo Mazzola, Department of Health Sciences, University of Milano-Bicocca, NeuroMI - Milan Center for Neuroscience, Geriatric Clinic, San Gerardo University Hospital, via Pergolesi 33, Floor 7 C, 20900 Monza (MB), Italy, Tel: +39 039-233-3475; Fax: +39 039-233-2220; E-mail: paolo.mazzola@unimib.it

Giorgio Annoni, Department of Health Sciences, University of Milano-Bicocca, NeuroMI - Milan Center for Neuroscience, Geriatric Clinic, San Gerardo University Hospital, via Pergolesi 33, Floor 7 C, 20900 Monza (MB), Italy, Tel: +39 039-233-3475; Fax: +39 039-233-2220; E-mail: giorgio.annoni@unimib.it

Key words: centenarians, hip fracture, orthogeriatric, multidisciplinary approach Received: May 11, 2015; Accepted: June 09, 2015; Published: June 12, 2015 
highest rate of increase. To date, about half of the Italian centenarians live in Northern Italy, which - along with Central Italy - is also the geographical area with the proportion of over 65 individuals?

Hip fracture (HF) is a frequent occurrence in the elderly, and its incidence grows exponentially as the population ages [4]. A number of studies have already described HF in the elderly, but the available literature focusing on centenarians is limited [2,5-8]. The present manuscript reviews the activity of our Orthogeriatric Unit (OGU) at San Gerardo University Hospital (Northern Italy), which is a reference center for hip fractures in Lombardy region $[9,10]$. We focused on centenarians admitted from 2007, when the OGU was established, until December, 2014. Our retrospective research prompted us to review the currently available literature, which revealed similar limitations.

\section{Methods}

\section{Inclusion and exclusion criteria}

We performed a retrospective review of the OGU activity at San Gerardo University Hospital, analyzing the medical records of all patients aged 100 years and older at the moment of admission, whose principal diagnosis was "hip fracture" or "proximal femur fracture". We identified 10 records of centenarian patients to review.

For the review of the literature currently available, we considered the English language papers published in PubMed online library, combining the key terms 'centenarian' and 'hip fracture,' or '100' and 'hip fracture'. We considered only the papers that discussed patients at least 100 years of age, and excluded all manuscripts not written in English. Based on these criteria, we identified 11 papers to review.

The study was approved by the Institutional Review Board of San Gerardo University Hospital.

\section{Centenarians admitted to the OGU: Review of the activity}

Since its establishment in 2007, the OGU has admitted 10 centenarians, all females. The characteristics of these patients are summarized in Table 1. The patients' mean age was $100.6 \pm 1.0$ years (range 100-103 years), while their average length of hospital stay was $14.1 \pm 6.0$ days. Four of the patients experienced pertrochanteric fractures, while the remaining six experienced femoral neck fractures. The mean Katz's ADL score [11] of the study population was $3.1 \pm 2.0$. All subjects underwent surgery. The characteristics of the patients' fractures and perioperative period are summarized in Table 2. Eight patients were assigned an American Society of Anesthesiologists physical status (A.S.A.) [12] score of 3, which means "severe systemic disease". ASA scores of 4 ("severe systemic disease that is a constant threat to life") and 2 ("mild systemic disease") were assigned in the remaining two cases. Following an evaluation within the OGU team, the orthopedic surgeons performed a hemiarthroplasty on two of the patients. In the remaining 8 cases, the surgeons performed an internal fixation ( 3 with screw-plate devices, 3 with cannulated screws, and 2 with gamma nail). In five out of ten surgical procedures, the anesthetist proposed general anesthesia which, in three cases, was combined with peripheral nerve block. Spinal anesthesia was adopted in three cases, and a loco-regional approach was performed twice. On average, centenarians were transfused with $1.6 \pm 1.2$ units of red blood cells (General transfusion index=units of $\mathrm{RBC} / \mathrm{No}$. of patients), although two patients whose fractures were fixed with cannulated screws did not require transfusions (Real transfusion index=units of $\mathrm{RBC} / \mathrm{No}$. of patients transfused=2.0). Only one centenarian experienced preoperative delirium, while four developed incident post-operative delirium, including the patient who developed it in the pre-operative phase.

According to our policy, seven patients were discharged to a rehabilitation facility, while two went directly home with a schedule of low-intensity physical therapy. We observed a single case of inhospital death. At the follow-up interview, we found that 2 patients died one month after discharge; the total number of deaths was 7 after 3 months (the same after 6 months) and 8 after one year. At the time of the manuscript preparation, two patients are still alive. The small sample size does not allow us to make reliable estimates of the mortality rate, but we confirm the high risk of postoperative death among centenarians undergoing hip fracture surgery $[2,5,6,8,13]$. Due to the overall short survival of the patients, which in one case was 14 days and in four cases were 40- 43 days following surgery, data regarding rehabilitation are difficult to retrieve. The patient (ID n. 1) who survived until 72 days post-operation died from progressive cachexia due to an occult malignancy of the uterus, without recovering her pre-operative autonomy. Patient n. 3 died 263 days after her operation, initially recovering her ability to walk but then gradually decaying from a cognitive and physical point of view, mainly due to a pelvic malignancy discovered during the OGU hospitalization. The two patients who are currently alive both recovered their ability to walk independently, the first (ID n. 5) with a stick and the second (ID n. 9) with a walker. Their quality of life, despite their need for assistance when bathing and dressing and their urinary incontinence, is perceived as acceptable. Neither residual pain of the operated hip nor further hospitalizations was reported by their caregivers. Overall, only 3 patients recovered their ability to walk within three months, whereas the other patients

Table 1. Case series: Demographic data and main characteristics of centenarian patients admitted to San Gerardo University Hospital (Monza, Italy) with a diagnosis of hip fracture, starting from the year of establishment of the Orthogeriatric Unit (2007).

\begin{tabular}{|c|c|c|c|c|c|c|c|c|c|}
\hline $\begin{array}{l}\text { Patient } \\
\text { No. }\end{array}$ & $\begin{array}{c}\text { Age } \\
\text { [years] }\end{array}$ & $\begin{array}{c}\text { Gender } \\
{[\mathbf{M} / \mathbf{F}]}\end{array}$ & \begin{tabular}{c|} 
Year of \\
hospitalization
\end{tabular} & $\begin{array}{l}\text { Pre-fracture } \\
\text { residence }\end{array}$ & Caregiver at home & $\begin{array}{l}\text { Katz's ADL } \\
\text { score }\end{array}$ & $\begin{array}{c}\text { Charlson Comorbidity } \\
\text { Index }\end{array}$ & $\begin{array}{l}\text { Medications } \\
{[\mathrm{n}]}\end{array}$ & $\begin{array}{c}\text { Preoperative delirium } \\
\text { [yes/no] }\end{array}$ \\
\hline 1 & 101 & $\mathrm{~F}$ & 2008 & Home & None & 4 & 10 & 7 & No \\
\hline 2 & 100 & $\mathrm{~F}$ & 2008 & Home & Family member & 2 & 5 & 4 & No \\
\hline 3 & 100 & $\mathrm{~F}$ & 2010 & Home & Family member & 4 & 1 & 1 & No \\
\hline 4 & 101 & $\mathrm{~F}$ & 2010 & Home & Family member & 2 & 4 & 7 & No \\
\hline 5 & 100 & F & 2011 & Home & Family member & 6 & 2 & 2 & No \\
\hline 6 & 103 & $\mathrm{~F}$ & 2011 & Home & Family member & 0 & 3 & 4 & No \\
\hline 7 & 101 & $\mathrm{~F}$ & 2012 & Home & Family member & 1 & 0 & 3 & Yes \\
\hline 8 & 100 & $\mathrm{~F}$ & 2012 & Home & Family member & 3 & 0 & 2 & No \\
\hline 9 & 100 & $\mathrm{~F}$ & 2013 & Home & Family member & 3 & 0 & 3 & No \\
\hline 10 & 100 & $\mathrm{~F}$ & 2014 & Home & None & 6 & 1 & 8 & No \\
\hline
\end{tabular}

*Activities of Daily Living. 
Table 2. Case series: Characteristics of fracture, surgical operation, and post-operative period of the centenarian hip fracture patients admitted to San Gerardo University Hospital (Monza, Italy), starting from the year of establishment of the Orthogeriatric Unit (2007).

\begin{tabular}{|c|c|c|c|c|c|c|c|c|c|}
\hline $\begin{array}{l}\text { Patient } \\
\text { No. }\end{array}$ & $\begin{array}{c}\text { ASA score } \\
{[1-5]}\end{array}$ & $\begin{array}{l}\text { Type of } \\
\text { fracture }\end{array}$ & Surgical treatment & Anesthesia & Transfusions [n] & $\begin{array}{c}\text { Postoperative delirium } \\
\text { [yes/no] }\end{array}$ & $\begin{array}{l}\operatorname{LOS}^{*} \\
\text { [days] }\end{array}$ & Discharge & $\begin{array}{c}\text { In-hospital mortality } \\
\text { [yes/no] }\end{array}$ \\
\hline 1 & 3 & FN & Hemiarthroplasty & General+PNB & 2 & Yes & 15 & Rehab & no \\
\hline 2 & 3 & PT & Screw-plate & General & 4 & No & 11 & Exitus & yes \\
\hline 3 & 3 & PT & Screw-plate & General+PNB & 2 & Yes & 22 & Rehab & no \\
\hline 4 & 3 & FN & Cannulated screws & General & 0 & No & 15 & Rehab & no \\
\hline 5 & 3 & FN & Hemiarthroplasty & Spinal & 1 & No & 15 & Rehab & no \\
\hline 6 & 4 & FN & Cannulated screws & General+PNB & 2 & Yes & 20 & Rehab & no \\
\hline 7 & 3 & PT & Screw-plate & Spinal & 2 & Yes & 7 & Rehab & no \\
\hline 8 & 3 & FN & Cannulated screws & Locoregional & 0 & No & 7 & Home & no \\
\hline 9 & 2 & FN & Gamma nail & Spinal & 1 & No & 7 & Rehab & no \\
\hline 10 & 3 & $\mathrm{PT}$ & Gamma nail & Locoregional & 2 & No & 22 & Rehab & no \\
\hline
\end{tabular}

* Length of stay. Abbreviations: FN: Femoral neck; PT: Pertrochanteric; PNB: Peripheral Nerve block; Rehab: Rehabilitation facility.

\section{died during this time frame.}

This analysis has limitations. First, in accordance with our aim, this is a single-center retrospective observational study, and therefore the results cannot be generalized. Second, the sample size is small and distributed over a long period (7 years). Despite the retrospective nature of the analyses, which is a common feature of the previous studies, a multicenter design would be desirable to increase the case series and to better analyze the outcomes of this expanding age group.

\section{Review of the literature}

The first study we found was a retrospective case series analysis of 13 subjects [5]. The study reviewed the local orthopedic activity of centenarians admitted to the Derbyshire Royal Infirmary (UK) between 1988-1999. As the authors expected, the study revealed high mortality rates, especially in-hospital mortality rates (4 cases, accounting for $31 \%$ of the study population). The mortality rates increased at 6 -month and 1 -year follow-ups, reaching $50 \%$ and $56 \%$, respectively. However, these results should be examined with consideration of the small sample size.

A second paper, by Oliver and colleagues, [2] compared 18 centenarians with a group of 18 "normal age HF patients", whose mean age was 78.2 years and who were randomly selected during a 5 -year period (1998-2002). They found that $28.6 \%$ of the centenarian patients were able to return to their homes, as opposed to a discharge toward a long-term facility, versus $69.2 \%$ of the normal age group. Mortality rates were reported to be similar to the study from Forster et al., [5] with an in-hospital mortality of $11.1 \%$, and 1- and 4-month mortality rates of $33.3 \%$ and $50 \%$, respectively.

In 2004, a study investigated whether centenarian hip fracture patients really need surgery and rehabilitation [3]. A retrospective case series analysis was performed on 23 patients in an Israeli teaching hospital during a 12-year study period (1990-2001). Nineteen of these subjects underwent surgery, with an average time to surgery of 2.23 days and a mean length of stay of 11.2 days. Mortality rates were $4.4 \%$ after 1 month, $34.8 \%$ after 6 months, $34.8 \%$ after 1 year, and $60.9 \%$ after 18 months. The authors suggest that surgery should not be denied based on extreme age, since they found that good functional outcomes and return to the pre-fracture state are achievable. They conclude that the decision of whether to perform hip fracture surgery and rehabilitation in centenarians should be determined on a case-by-case basis.

Another case series analysis was described by Konttinen et al. in Finland [1]. The authors focused on 12 cases of centenarians that underwent major emergency surgery. In this retrospective analysis from 1990 to 2004, 9 out of 12 subjects were admitted to their hospital for hip fracture. One-month mortality rate was $25 \%, 6$-month mortality rate was $42 \%$, and 1 -year mortality rate was $50 \%$. The authors conclude that regardless of the type of anesthesia used, episodes of marked hypotension requiring pharmacological correction were common among centenarians undergoing emergency surgery. They suggest that hypovolemia may explain the high hemodynamic instability seen in these patients.

In response to this paper, a letter from Eipe and colleagues [14] described the case of a centenarian patient from rural India who received marked benefits from HF surgery, despite 4 weeks of an unsuccessful conservative approach. The authors underline the importance of appropriate counseling, a well orchestrated multidisciplinary approach, and a follow-up appointment in order to obtain positive patient outcomes.

In 2009, a manuscript from Kent and colleagues described the results of a retrospective observational database analysis (2004-2005) in a UK hospital in Dorset [15]. The authors focused on 26 trauma admissions in centenarians, 13 of whom were hospitalized for HF. They found in-hospital mortality to reach $19.2 \%$, while 1-month and 1-year mortality rates were $33 \%$ and $42 \%$, respectively. They conclude that centenarians should be afforded the same consideration for surgery as younger subjects.

An article from Verma et al., [6] also analyzing the activity of a UK hospital, compared length of stay and time to surgery in centenarians versus younger subjects to investigate whether the oldest patients require additional costs for their treatment. Twenty-six centenarians and 50 elderly patients (mean age $=80.5$ years) were identified. The mean time to surgery was 3.6 days for centenarians and 1.9 days for the younger ones (non-significant), and the average length of stay was 20.7 days versus 14.9 days, respectively $(\mathrm{p}=0.0015)$. In-hospital mortality rate was $17.3 \%$ for centenarians $v$ s. $2 \%$ for elderly patients, and 1 -month mortality rate was $30.4 \%$ for centenarians $v$ s. $4.1 \%$ for elderly patients. The authors estimate that centenarians undergoing hip fracture surgery require an average additional cost of $£ 2511$ (adjusted: $£ 2208$ ).

Referring to the period from 2001 to 2006, Pelavski and colleagues [16] published a retrospective review of activity in a teaching hospital in Barcelona, Spain, focusing on the transfusion practice during hospitalization. They compared 17 centenarian hip fracture patients with 17 "typical age range patients" (mean age $=77$ years) randomly selected from their database. The significant differences they reported 
were postoperative hemoglobin levels, which were higher for the "typical age range" group, trigger hemoglobin levels, and the incidence of postoperative complications, both of which they found to be higher for centenarians. The in-hospital mortality rate was $11.8 \%$ among centenarians, while no cases of death were reported in the typical age range group.

In 2010, a letter from Rodriguez-Molinero and colleagues [7] reported an observational analysis of the Spanish hospitalization database, focusing on centenarians admitted for HF during 2005. They received a total of 162 centenarian HF patients, and found that HF was the leading cause of hospitalization in this segment of the population. In-hospital mortality rate was $17.3 \%$, and the estimated costs related to the care of centenarians, particularly indirect costs, were high. To the best of our knowledge, this is the only study that uses a national database to focus on HF in centenarians.

More recently, Patil et al. [13] published a retrospective review of the 2000-2009 activity of a US hospital in Livingston, New Jersey. The authors described 13 centenarian hip fracture patients, 12 of whom underwent surgery. Five patients $(41.7 \%)$ were transfused in the perioperative phase. One patient (7.7\%) died during hospitalization due to severe pneumonia, which progressed to multisystem organ failure. Two other patients developed sacral decubitus ulcers. According to their database, HF accounted for more than $10 \%$ of the hospitalizations among centenarians and $29 \%$ of all of the surgeries performed in this age group. They highlight that HF surgery is well tolerated in centenarians, and thus it should be suggested regardless of their extreme age.

Finally, in 2013, Tarity et al. [8] published a retrospective review of the 2003-2010 activity at Thomas Jefferson University Hospital in Philadelphia. The authors described a case series of 23 centenarian patients, 21 of whom underwent hip fracture surgery (mean time to surgery was 0.9 days). Their analysis was oriented towards the longterm follow-up. The patients showed an in-hospital mortality rate of $15 \%$. Cumulative mortality was $20 \%$ after 1 month, $30 \%$ after 3 months, $45 \%$ after 6 months, $60 \%$ at the 1 -year follow-up, $70 \%$ after 2 years, and $90 \%$ after 3 years.

\section{Discussion}

According with estimates by the ISTAT, the proportion of elderly subjects continues to increase in Italy, and centenarians show the highest rate of increase (estimated $+14.5 \%$ from 2013 to 2015, available at http://demo.istat.it/uniprev2011/index.html).

$\mathrm{HF}$, one of the most frequent age-related injuries, is an important cause of hospitalization among centenarians $[7,13,15]$. While the inhospital mortality is between $7.7 \%-31 \%$, as reported by the literature, the short- and medium-term mortality rates are particularly high in this group of extreme age $[2,5-8,13]$. The findings of this retrospective analysis of the centenarians admitted to our OGU are consistent with the existing literature in terms of both in-hospital and 1-year mortality rates. To date, two patients were diagnosed with cancer after hip fracture, and their deaths were a result of these severe comorbid conditions, rather than of complications related to HF itself.

Globally, possible explanations for the high mortality rates seen in centenarians with HF include their remaining lifespan, chronic comorbidity, and functional disability. Chronic comorbidity is a common feature of centenarians, and since it is associated with a very limited organ reserve, it often contributes to the extreme frailty of these patients. Among the oldest old (i.e. people aged $\geq 85$ years), functional disability is common both pre-operatively and as a result of failure in post-operative rehabilitation following hip fracture surgery, and represents a risk factor for poor survival $[17,18]$. Furthermore, comorbidity and limited organ reserve frequently lead to short-term postoperative complications following emergency surgery, in particular to cardiovascular complications such as hypotension [1]. The common occurrence of delirium and pulmonary complications in centenarians further reflects their frailty status.

However, neither age per se, nor pre-fracture mobility impairment should exclude centenarians from the option to undergo surgery, since their mortality risk is overshadowed by the potential benefits following fixation. Indeed, a few studies report extremely poor clinical outcomes for those patients treated conservatively, in terms of pain, complications, and mortality rate $[3,8]$. If the rates of recovery are low in terms of pre-fracture walking ability and living status, we must take into account that all of the studies to date have been performed on small samples. When comparing a surgical versus a conservative approach, hip surgery leads to better pain control and early mobilization, which are essential determinants of quality of life. However, a proper analysis of the outcomes of HF surgery and rehabilitation in centenarians, as well as its cost-effectiveness, is currently not achievable due to several limitations.

\section{Limitations}

The studies currently available are too sparse and take place over a long period of time. The majority of them describes case series of small sample size and use only a single center, except for one study which described the incidence of HF in the Spanish population using a national on-line database and 162 subjects [7]. In addition, all of the studies adopted a retrospective design. For all of the above reasons, their results cannot be generalized.

Another limitation of the current studies is the heterogeneity of their objectives and methods, including the different time-points adopted for the follow-up appointments. Finally, the research papers rarely include data regarding direct costs sustained for the hospitalization and procedures.

This research area would be improved by expanding the size of the study populations, possibly by implementing regional or national hip fracture databases. Data collection should be designed in a prospective fashion, pursuing uniformity of the in-hospital and follow-up outcome measures.

In a period of financial crisis, inclusion of the calculation of direct costs would allow critical analyses of cost-effectiveness focused on hospital admissions in centenarians, especially for those undergoing surgical procedures such as hip fracture surgery.

Multicenter national and international data exchange will allow for proper future research oriented towards risk/benefits of the hospital procedures, cost-effectiveness, and quality of life optimization in centenarians.

\section{Conclusion}

Despite centenarians representing a small proportion of the elderly patients requiring hospitalization, they are the age group with the fastest estimated growth. Consequently, the prevalence of centenarians' hospital admissions and the absolute number of hip fractures seen in hospitals will increase. This will pose a significant challenge to physicians and surgeons, who will need to focus more and more on quality of life in a population with limited remaining lifespan. Although a complete 
recovery of the pre-fracture functional status is undoubtedly difficult among centenarians, early surgical fixation and mobilization should still be recommended for the preservation of quality of life, and to potentially increase the rates of restoration of pre-fracture living and ambulatory status. At present, a shift from the "single specialty" care toward a multidisciplinary management is necessary in this extreme age group and in the elderly in general. Finally, in line with the need of large-scale datasets, we are developing a collaboration with the Lombardy Region to realize a study that will enlighten the real-world burden of hip fracture in centenarians through the National Healthcare Utilization database.

\section{Acknowledgments}

PM, FDF, MC, GC, GB, GZ, and GA took care of the patients and collected the informed consent forms. PM, GB, GA: study design. PM, DP, FDF, GC, ET: data collection from the medical records. PM, SP, DP, ET, MC, FDF, GC: review of the literature and manuscript preparation. GA, GB, PM, GZ: critical revision of the manuscript and revision of the English version.

\section{References}

1. Konttinen N, Rosenberg PH (2006) Outcome after anaesthesia and emergency surgery in patients over 100 years old. Acta Anaesthesiol Scand 50: 283-289. [Crossref]

2. Oliver CW, Burke C (2004) Hip fractures in centenarians. Injury 35: 1025-1030. [Crossref]

3. Shabat S, Mann G, Gepstein R, Fredman B, Folman Y, et al. (2004) Operative treatment for hip fractures in patients 100 years of age and older: is it justified? J Orthop Trauma 18: 431-435. [Crossref]

4. Samelson EJ, Zhang Y, Kiel DP, Hannan MT, Felson DT (2002) Effect of birth cohort on risk of hip fracture: age-specific incidence rates in the Framingham Study. Am J Public Health 92: 858-862. [Crossref]

5. Forster MC, Calthorpe D (2000) Mortality following surgery for proximal femoral fractures in centenarians. Injury 31: 537-539. [Crossref]
6. Verma R, Rigby AS, Shaw CJ, Mohsen A (2009) Acute care of hip fractures in centenarians--do we need more resources? Injury 40: 368-370. [Crossref]

7. Rodríguez-Molinero A, Yuste A, Banegas JR (2010) High incidence of hip fracture in Spanish centenarians. J Am Geriatr Soc 58: 403-405. [Crossref]

8. Tarity TD, Smith EB, Dolan K, Rasouli MR, Maltenfort MG (2013) Mortality in centenarians with hip fractures. Orthopedics 36: e282-287. [Crossref]

9. Mazzola P, De Filippi F, Castoldi G, Galetti P, Zatti G, et al. (2011) A comparison between two co-managed geriatric programmes for hip fractured elderly patients. Aging Clin Exp Res 23: 431-436. [Crossref]

10. Bellelli G, Mazzola P, Corsi M, Zambon A, Corrao G, et al. (2012) The combined effect of ADL impairment and delay in time from fracture to surgery on 12-month mortality: an observational study in orthogeriatric patients. J Am Med Dir Assoc 13: 664. [Crossref]

11. Katz S, Downs TD, Cash HR, Grotz RC (1970) Progress in development of the index of ADL. Gerontologist 10: 20-30. [Crossref]

12. Owens WD, Felts JA, Spitznagel EL Jr (1978) ASA physical status classifications: a study of consistency of ratings. Anesthesiology 49: 239-243. [Crossref]

13. Patil S, Parcells, B, Balsted, A, Chamberlain, RS (2012) Surgical outcome following hip fracture in patients $>100$ years old: will they ever walk again? Surgical Science 3: 554-559.

14. Eipe N, Padhi P, Padhi NR (2006) Management of centenarian hip fractures. Acta Anaesthesiol Scand 50: 902-904. [Crossref]

15. Kent MJ, Elliot RR, Taylor HP (2009) Outcomes of trauma in centenarians. Injury 40 358-361. [Crossref]

16. Pelavski Atlas AD, Colomina MJ, De Miguel M, Roige J (2009) Centenarians versus patients within the most frequent age range for hip fractures: transfusion practice. Arch Orthop Trauma Surg 129: 685-689. [Crossref]

17. Mazzola P, Bellelli G, Broggini V, Anzuini A, Corsi M, et al. (2015) Postoperative delirium and pre-fracture disability predict 6-month mortality among the oldest old hip fracture patients. Aging Clin Exp Res 27: 53-60. [Crossref]

18. Torpilliesi T, Bellelli G, Morghen S, Gentile S, Ricci E, et al. (2012) Outcomes of nonagenarian patients after rehabilitation following hip fracture surgery. $\mathrm{J} \mathrm{Am} \mathrm{Med} \mathrm{Dir}$ Assoc 13: 81. [Crossref]

Copyright: (C2015 Mazzola P. This is an open-access article distributed under the terms of the Creative Commons Attribution License, which permits unrestricted use, distribution, and reproduction in any medium, provided the original author and source are credited. 